\title{
Searching for modifications to the exponential radioactive decay law with the Cassini spacecraft
}

\author{
Peter. S. Cooper \\ Fermi National Accelerator Laboratory, Batavia, IL 60510, U.S.A.
}

(Dated: October 24, 2018)

\begin{abstract}
Data from the power output of the radioisotope thermoelectric generators aboard the Cassini spacecraft are used to test the conjecture that small deviations observed in terrestrial measurements of the exponential radioactive decay law are correlated with the Earth-Sun distance. No significant deviations from exponential decay are observed over a range of $0.7-1.6 A$.U. A $90 \% \mathrm{Cl}$ upper limit of $0.84 \times 10^{-4}$ is set on a term in the decay rate of ${ }^{238} \mathrm{Pu}$ proportional to $1 / R^{2}$ and $0.99 \times 10^{-4}$ for a term proportional to $1 / R$.
\end{abstract}

PACS numbers: 23.60.+e, 23.40.-s, 95.55.Pe, 96.60.Vg, 0620.Jr.

A recent archive preprint reports evidence for a correlation between nuclear decay rates and the Earth-Sun distance 1]. This correlation is extracted from an annual modulation in the observed decay rates of ${ }^{32} \mathrm{Si} /{ }^{36} \mathrm{Cl}$, both $\beta$ emitters, and ${ }^{226} R a$, an $\alpha$ emitter. Reference 1 analyzes this as a correlation with $1 / R^{2}(t)$, the variation in the Earth-Sun distance due to the eccentricity of the Earth's orbit. To set a scale for this correlation the amplitude of the decay rate variation is $\sim 0.1 \%$ roughly in phase with the $3 \%$ annual modulation in $1 / R^{2}(t)$ suggesting a $\left(3 \times 10^{-2}\right) / R^{2}$ term in the decay rate.

In the conclusion of this paper the authors observe that: These conclusions can be tested... [by] measurements on radioactive samples carried aboard spacecraft to other planets [which] would be very useful since the sample-Sun distance would vary over a much wider range. I report here the results of exactly such a measurement based on the power output of the Radioisotope Thermoelectric Generators (RTG) aboard the Cassini spacecraft which launched in 1997 and reached Saturn in 2004.

Cassini is powered by three RTGs each of which is a very large $(7.7 \mathrm{Kg}, 130 \mathrm{KCu}){ }^{238} \mathrm{Pu}$ radioactive source, an $\alpha$ emitter with an $87.7 y$ half life 2]. The heat from these sources are converted to electric power with thermoelectric piles. Together these sources produced $878 \mathrm{w}$ of electrical power from $\sim 13 \mathrm{Kw}$ of radioactive decay heat at launch. The power output of these RTGs were literally the lifeblood of the Cassini mission. Their power output was monitored carefully and often.

The trajectory of the Cassini spacecraft is available on the web [3]. I've used these data, converted to astronomical units $(A . U$.$) , to compute 1 / R(t)^{2}=R_{e}^{2} /\left[x(t)^{2}+\right.$ $\left.y(t)^{2}+z(t)^{2}\right]$. Over the first 2 years Cassini went from $R(0)=1$ at launch, made 2 visits to Venus at $R=0.7$ and crossed the orbit of the earth a total of 4 times before finally gaining enough speed to reach Saturn at $\mathrm{R}=9$.

JPL kindly provided [4] $\mathrm{P}(\mathrm{t})$, the total electrical power from the three RTGs aboard, measured daily since launch and the expected power output from their RTG modeling. The distance of Cassini from the Sun and the



FIG. 1: Left, suppressed zero, scale: solid red curve, Heliocentric distance $[R(t)(A . U)$.$] , Black points are the 5$ times when $\mathrm{R}(\mathrm{t})=1$ (green line), Right, suppressed zero, scale: blue diamonds, RTG electrical power.

electrical power output are plotted in Figure 1 for the first 2 years of the mission. $\mathrm{R}$ ranged from $0.7-1.6$ A.U. $\left(0.35<1 / R^{2}<2.2\right)$. The power dropped from $878 w$ at launch to $815 \mathrm{w}$ over this period.

The thermal power output of an RTG is directly proportional to the decay rate of the radioisotope generating the heat: $P_{t h}(t)=N_{0} \lambda \exp (-\lambda t) E_{d}$, where $E_{d}$ is the energy released per decay. The electrical power output, $P(t)=P_{t h}(t) \epsilon_{0} \epsilon(t)$ is modified by an initial efficiency, $\epsilon_{0}$, and a time (or power) dependent thermoelectric conversion efficiency; $\epsilon(t), \epsilon(0)=1$.

I cannot safely use the RTG efficiency model[5] here since it assumed only exponential behavior for radioac- 


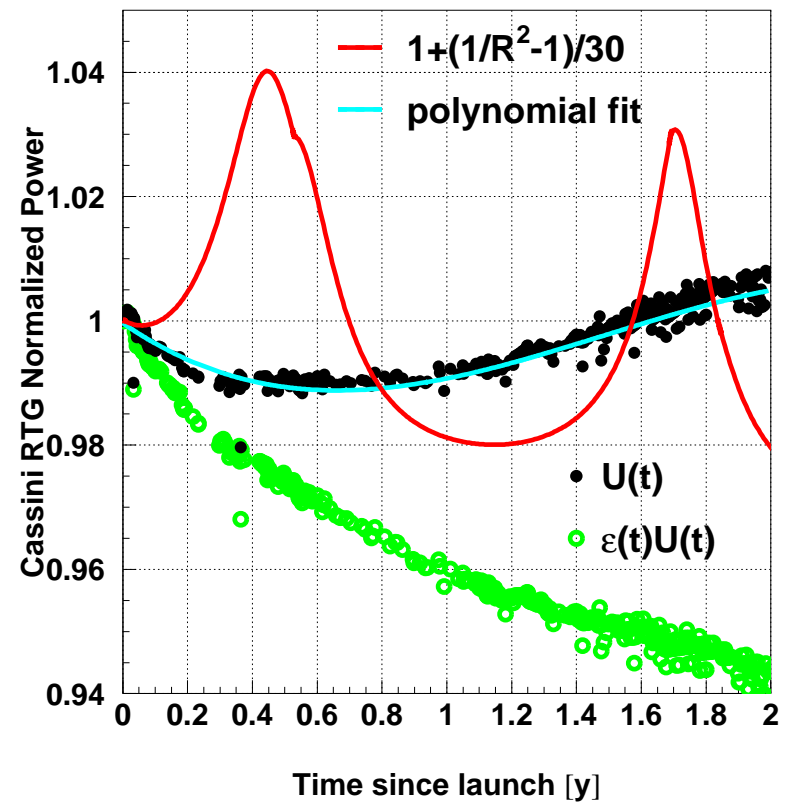

FIG. 2: RTG power; green open circles, decay corrected $[\epsilon(t) U(t)]$, black solid circles, efficiency and decay corrected $[U(t)]$, cyan curve, 3rd order polynomial correction function described the in text. red solid line, expected effect extrapolated from Reference 1: $1-\left(1 / R^{2}(t)-1\right) / 30$.

tive decay. Any new physics effect might be inadvertently subsumed into that model. The Cassini trajectory provides a natural calibration for $\epsilon(t)$ using the measured power at the 5 points (shown in Figure 1) where the spacecraft was $1 A . U$. from the sun. Fitting these measurements to $P_{R=1}(t)=P(0) 2^{(-t / 87.7 y)} 2^{\left(-t / T_{e f f}\right)}$ yields $T_{\text {eff }}=21.2 \pm 1.9 y$. This simple model agrees in shape with Reference 4 and obeys the requirements of Carnot thermal efficiency: as the power decreases, and the temperature difference across the thermoelectric piles decrease, the efficiency can only decrease. Something as complicated as a space-born thermoelectric pile requires more than one parameter to accurately describe its behavior. The single exponential reduces a $5 \%$ power drop in the first 2 years to a $\pm 1 \%$ variation from unity.

Following Reference 1 I've plotted $\epsilon(t) U(t)=$ $P(t) / P(0) 2^{t / 87.7 y}$, the normalized electrical power corrected for ${ }^{238} P u$ decay, and $\mathrm{U}(\mathrm{t})$, the normalized thermal power, as a functions of time since launch in Figure 2 for the first 2 years of Cassini's voyage to Saturn.

To compare to the rough magnitude of the effect reported by reference 1 I've plotted $U_{\text {ref } 1}(t)=1+$ $(0.1 \% / 3 \%)\left(1 / R(t)^{2}-1\right)$ on Figure 2 to extrapolate the small $\mathrm{R}$ variation of reference 1 for comparison with the larger $\mathrm{R}$ range available in this study. Extrapolating a $0.1 \%$ decay rate change for a $3 \%$ change in $1 / R^{2}$ to a $50 \%$ change in $1 / R^{2}(R=0.7 A . U ., t=0.43 y)$ should cause a

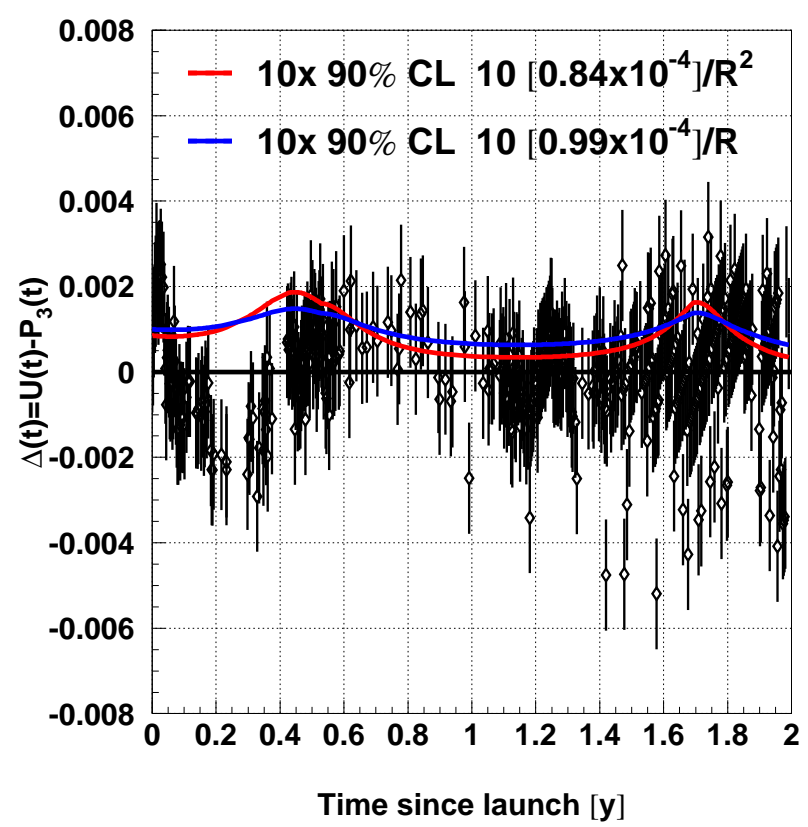

FIG. 3: Black points; $\Delta(t)$, RTG normalized thermal power less a 3rd order polynomial correction, Red curve; 10 times the $90 \%$ CL limit, $10 \alpha / R^{2}$, Blue curve; 10 times the $90 \% \mathrm{CL}$ limit, $10 \beta / R$.

$+4 \%$ change in the power output of the RTG 5 months after launch. In fact $U(t)$ decreases by $\sim 1 \%$. Changes this large are excluded by the Cassini efficiency corrected data both in magnitude and by the absence of any reflection of the shape of $1 / R^{2}(t)$ in either normalized power curve.

In order to set quantitative limits I've fit the efficiency and half-life corrected $U(t)$ normalized power data (suppressing the two obvious outlying points in Figure 1) to $P_{3}(t)$, a 3 th order polynomial in time, to phenomenologically describe the last $1 \%$ variation in the $U(t)$. As shown in Figure 2, this polynomial smoothly interpolates the $U(t)$ measurements. A 3th order polynomial make a very poor fit to $1 / R^{2}(t)$; these two shapes are approximately orthogonal. The error assigned to each $\mathrm{U}(\mathrm{t})$ measurement by requiring this fit to have $\chi^{2} / \nu=1$ is 0.0015 . The individual relative power measurements have a resolution of $0.15 \%$ and the polynomial is a $\sim 6 \sigma$ systematic correction beyond the simple exponential efficiency model.

The difference of the data from the polynomial fit; $\Delta(t)=U(t)-P_{3}(t)$ are plotted in Figure 3. Some structure at the $1 \sigma$ level and some outlying measurements remain. These difference data are fit to $\alpha / R^{2}$ and $\beta / R$ to give limits on the contribution of a term in the ${ }^{238} P u$ decay rate dependent on the Earth-Sun distance. The $90 \% C L$ limit on from these fits are $|\alpha|<0.84 \times 10^{-4}$ and $|\beta|<0.99 \times 10^{-4}$ respectively. The limiting functions, 
scale up by a factor of 10 for visibility, are also shown in Figure 3. $\alpha$ is to be compared with the correlation seen in Reference 1 for ${ }^{226} R n$ decay of $\sim+3 \times 10^{-2}$.

The Cassini RTG power data exclude any variation of the ${ }^{238} \mathrm{Pu}$ nuclear decay rate correlated with the distance of the source from the Sun to a level $350 \times$ smaller than the effect reported by Reference $1 .{ }^{238} \mathrm{Pu}$ and ${ }^{226} \mathrm{Ra}$ are similar $\alpha$ emitters. Another physical or experimental cause of the reported annual variations in nuclear decay rates appears to be necessary. More generally Rutherford, Chadwick, and Ellis's 1930 conclusion that The rate of transformation of an element has been found to be constant under all conditions. [6] now has solid experimental support at least from Venus $(\mathrm{R}=0.7)$ to Mars $(\mathrm{R}=1.5)$.

I am indebted to several of my colleagues for calling this paper and physics issue to my attention; Chris Quigg and Martin Hu of Fermilab and Jurgen Engelfried of the Universidad Autǿnoma de San Luis Potosí, Mexico. I am also thankful for very useful lunchtime conversations on this subject with several of my Fermilab colleagues. I an indebted to Richard Ewell and Torrence Johnson of JPL for making the RTG data available and Stephen Parke of Fermilab for critical comments on this manuscript.

[1] Jere H. Jenkins et al., Evidence for Correlations Between Nuclear Decay Rates and the Earth-Sun Distance, arXiv:astro-ph:0808.3283v1.

[2] National Nuclear Data Center, http://www.nndc.bnl.gov/

[3] http://www.lepp.cornell.edu/ seb/celestia/cassini-all.zip

[4] private communication, R. Ewell, JPL.

[5] R. Ewell, D. Hanks, J. Lozano, V. Shields, \& E. Wood, DEGRA - A computer Model for Predicting Long Term Thermoelectric Generator Performance, Space Technologies and Applications International Forum (STAIF), Albuquerque, New Mexico, February 12-16, 2005., http://hdl.handle.net/2014/38760

[6] S. E. Rutherford, J. Chadwick, and C. Ellis, Radiations from Radioactive Substances (Cambridge University Press, 1930). 